\title{
Structural metrics of high-temperature lattice conductivity
}

\author{
B. L. Huang and M. Kaviany ${ }^{\text {a) }}$ \\ Department of Mechanical Engineering, University of Michigan, Ann Arbor, Michigan 48109-2125
}

(Received 10 July 2006; accepted 14 September 2006; published online 21 December 2006)

\begin{abstract}
An atomic structure-based model for high-temperature lattice conductivity is developed for both compact crystals and cage-bridge crystals. For compact crystals, where long-range acoustic phonons dominate, the Debye temperature $T_{D}$ and Grüneisen parameter $\gamma$ are estimated using interatomic potentials to arrive at the lattice conductivity relation. Under the assumption of homogeneous deformation, $T_{D}$ is estimated according to a simplified force constant matrix and a phenomenological combinative rule for force constants, which is applicable to an arbitrary pair of interacting atoms. Also, $\gamma$ is estimated from a general Lennard-Jones potential form and the combination of the bonds. The results predicted by the model are in close agreement with the experimental results. For cage-bridge crystals, where both short-range acoustic phonons and optical phonons may be important, a simple mean-free path model is proposed: The phonon mean-free path of such a crystal at high temperatures is essentially limited by its structure and is equal to the cage size. This model also shows good agreement with the results of experiments and molecular dynamics simulations. Based on this atomic-level model, the structural metrics of crystals with low or high lattice conductivity are discussed, and some strategies for thermal design and management are suggested. (C) 2006 American Institute of Physics. [DOI: 10.1063/1.2396794]
\end{abstract}

\section{INTRODUCTION}

The lattice thermal conductivities of crystals are of practical and theoretical importance. They are essential for the reliability and performance of energy conversion systems, thermal insulators and conductors, microdevices, and microelectronic systems. For industrial applications, lattice conductivity is often obtained by the measurements of bulk crystals. But some crystals, such as zeolites, cannot be grown large enough for a direct measurement, and their thermal conductivities are often extracted from indirect measurements with large uncertainty. ${ }^{1}$ Furthermore, to synthesize a new material with the desired thermal properties, or to improve the performance of an existing material, a fundamental understanding of thermal transport in the crystals and its relation to the atomic structure is needed. An approach that can readily estimate the thermal behavior of a crystal with acceptable accuracy is especially useful.

The common approaches for predicting the lattice conductivity of a crystal fall into two categories, both of which are based on the Fourier law. One is to use an atomistic technique, such as molecular dynamics (MD) simulations. $\mathrm{MD}$ requires only the inputs of the configuration of atoms and suitable interatomic potentials. It is in principle a very fundamental method and often used as a tool for understanding thermal transport, in some cases with good agreement with experiments (at relatively high temperatures). ${ }^{2-4}$ However, accurate potentials are vital for MD, and the potentials used in MD are often obtained by fitting to ab initio calculations or experimental results, which is often very difficult, especially for complex polyatomic crystals. ${ }^{5}$ Also, MD is often very cumbersome and time-consuming, and the numerical results cannot provide much explicit information for

${ }^{a)}$ Electronic mail: kaviany@umich.edu the structure design and optimization. The other strategy for predicting the thermal conductivity is to use the continuum transport theory and the kinetic theory, such as the Boltzmann transport equation (BTE) approach of Callaway ${ }^{6}$ and Holland. $^{7}$ These approaches use the relaxation time approximation and can be used to calculate lattice conductivities quickly, but they require a good understanding of the underlying phonon scattering processes. Many such approaches have some parameters that must be obtained empirically, e.g., by fitting to the experimental or MD results, ${ }^{6-8}$ and are, therefore, not suitable for the prediction or design of new materials.

Starting from the derivation by Julian, ${ }^{9}$ in which the variational principle is used to obtain an analytical relaxation time for the rare-gas solids, Slack proposed that when heat is mainly carried by acoustic phonons scattered via the threephonon process, the thermal conductivity of crystals with constant volume at high temperatures (normally above $1 / 4-1 / 5$ of the Debye temperature) can be given by the relation $^{10,11}$

$$
k=\frac{3.1 \times 10^{4}\langle M\rangle \delta T_{D, \infty}^{3}}{T\left\langle\gamma^{2}\right\rangle N_{c}^{2 / 3}} .
$$

Here $\langle M\rangle$ is the mean atomic weight of the atoms in the primitive cell, $\delta^{3}$ is the average volume per atom, $T_{D, \infty}$ is the high-temperature Debye temperature, $T$ is the temperature, $N_{c}$ is the number of atoms in a primitive cell, and $\left\langle\gamma^{2}\right\rangle$ is the mode-averaged square of the Grüneisen parameter at high temperatures. Note that $T_{D, \infty}$ used by Slack is extracted from the phonon density of states (DOS) $D_{p},{ }^{11}$ i.e., 


$$
T_{D, \infty}^{2}=\frac{5 h^{2}}{3 k_{B}^{2}} \frac{\int_{0}^{\infty} \nu^{2} D_{p}(\nu) \mathrm{d} \nu}{\int_{0}^{\infty} D_{p}(\nu) \mathrm{d} \nu},
$$

where $h$ is the Planck constant, $k_{B}$ is the Boltzmann constant, and $\nu$ is the phonon frequency. However, $T_{D, \infty}$ cannot be conveniently determined for it requires the information of DOS. Since the difference between $T_{D, \infty}$ and the Debye temperature $T_{D}$ at $0 \mathrm{~K}$ ) extracted from the elastic constant or the measurement of heat capacity is normally small, it is customary to use $T_{D}$ instead of $T_{D, \infty}$ in Eq. (1). Also $\left\langle\gamma^{2}\right\rangle$ is often replaced by $\langle\gamma\rangle^{2}$ (later, for simplicity we use $\gamma$ to denote $\langle\gamma\rangle)$, which can be determined from thermal expansion data at high temperatures.

Equation (1) is widely tested with pure nonmetallic crystals and the overall agreement is good, even for complex crystals. ${ }^{10,11}$ The Slack relation illuminates how the atomic structure affects the thermal transport and provides a useful guide to tailoring the thermal transport properties.

McGaughey and Kaviany ${ }^{2}$ showed that the lattice thermal conductivity can be decomposed into three parts:

$$
k=k_{l g, A}+k_{\mathrm{sh}, A}+k_{O} .
$$

Here $k_{l g, A}$ is the contribution from long-range acoustic phonons, whose mean-free path is larger than one-half of their wavelength; $k_{\mathrm{sh}, A}$ is the contribution from short-range acoustic phonons, whose mean-free path is minimized to one-half of their wavelength; and $k_{O}$ is the contribution from optical phonons. Our previous work, ${ }^{12}$ has pointed out that the Slack relation corresponds to $k_{l g, A}$ and is valid only when the short-range acoustic or optical phonons are not important. This condition is not always satisfied for crystals with low thermal conductivity, such as zeolites and metal-organic frameworks (MOFs). To identify the relative contributions of these different mechanisms, a critical angular frequency $\omega_{c}$ based on Klemen's model ${ }^{12}$ is used, which is given as ${ }^{12}$

$$
\omega_{c} \simeq \frac{2.37 \times 10^{-27}\langle M\rangle u_{p, g}^{3}}{3 \pi^{2} \delta \gamma^{2} k_{B} T}
$$

where $u_{p, g}$ is the average phonon group velocity. The Slack relation is valid only when $\omega_{c}$ is comparable with the Debye frequency $\omega_{D}$ and long-range acoustic phonons dominate the thermal transport.

To use the Slack relation, $T_{D}$ and $\gamma$ must be known, which is the main difficulty in the estimation of the lattice conductivities of new materials. Since these two parameters directly relate the atomic structure to thermal transport, the knowledge of their relations provides more insightful information for the thermal design, and allows for the estimation of thermal transport properties of new materials.

In this work, we report a simple microscopic model to estimate $T_{D}$ and $\gamma$. A phenomenological combinative rule for force constants is proposed, which can be used to derive the force constant of an arbitrary pair bond from the existing experimental data. Then a model for the phonon group velocity and the Debye temperature of complex crystals is derived on the basis of the dynamical matrix, in which the effects of the lattice and bases are decomposed by the equivalent force constant. Using a general potential form for a bond, the Grüneisen parameters of different types of bonds are discussed, and then a relation for the equivalent Grüneisen parameter of the equivalent bond is developed, which can be applied for complex crystals. For cage-bridge structures, where the Slack relation may not be applicable, a simple phonon mean-free path model based on the kinetic theory is also proposed, which shows good agreement with experiments and MD.

\section{COMBINATIVE RULE FOR FORCE CONSTANTS OF AN ARBITRARY PAIR-BOND}

The vibration energy is transferred in a crystal through interactions among the atoms, which can be theoretically calculated by quantum mechanical methods. However, a quantum mechanical method deals with the electron clouds of the atoms, and is very cumbersome for a system involving many particles. Based on the Born-Oppenheimer approximation, ${ }^{5}$ the force field method uses empirical potentials (fitted to experiments or quantum mechanic calculations), such as Lennard-Jones and Buckingham potentials, to describe the interactions in the system. In most solids, when the temperature is well below the melting point, the particles only slightly oscillate around their equilibrium positions and many of their behaviors (including the elastic behavior) can be well described in the framework of the harmonic approximation. ${ }^{13}$ In this approximation, the energy of the system can normally be decomposed into four terms corresponding to the bond stretching, bending, torsion, and the nonbonded interactions, ${ }^{5}$ i.e.,

$$
\begin{aligned}
E= & \sum_{i} \frac{K_{i}}{2}\left(\Delta r_{i}\right)^{2}+\sum_{j} \frac{K_{\theta, j}}{2}\left(\Delta \theta_{j}\right)^{2}+\sum_{l} \frac{K_{\phi, l}}{2}\left(\Delta \phi_{l}\right)^{2} \\
& +\sum_{n} \frac{K_{m, n}}{2}\left(\Delta r_{m, n}\right)^{2},
\end{aligned}
$$

where $K, K_{\theta}, K_{\phi}$, and $K_{m, n}$ are the force constants of the bond length $r$, bond angle $\theta$, torsion angle $\phi$, and the distance between molecules $r_{m}$. Normally, the stretching interaction is much stronger than the other interactions (by a factor of more than 10), so for a rigid structure, the elastic characteristics are mainly determined by the stretching force constants. The bending and torsion interactions are also important for structure stability and deformation.

Since atomic interaction is determined by the electronic structure, potentials and force constants are expected to be transferable if the bond type and surroundings are similar. ${ }^{14}$ Here we present a phenomenological combinative rule for the stretching and the van der Waals force constants.

The general form of two-body potentials can be written as

$$
\phi_{A B}(r)=\varphi_{A B, \text { rep }}(r)-\varphi_{A B, \text { att }}(r),
$$

where $\varphi_{A B}$ is the potential energy of the bond $A-B$, and the subscripts rep and att represent the repulsive and the attractive terms. The repulsive term is due to the Pauli exclusion principle or the electrostatic interactions. It has been shown that the exchange repulsive term for two different atoms can 
be given as the geometric mean of the corresponding terms for two pairs of equivalent atoms, ${ }^{15}$ i.e.,

$$
\varphi_{A B, \text { rep }}(r)=\left[\varphi_{A A, \text { rep }}(r) \varphi_{B B, \text { rep }}(r)\right]^{1 / 2} .
$$

The attractive term is due to the interactions of dipoles, electrostatics, or a combination of them. The exchangeability of the dipolar and electrostatic interactions is apparent, thus a similar combinative rule is suggested for the attractive term, i.e.,

$$
\varphi_{A B, \mathrm{att}}(r)=\left[\varphi_{A A, \mathrm{att}}(r) \varphi_{B B, \mathrm{att}}(r)\right]^{1 / 2} .
$$

The potential near the equilibrium position can be described by the general Lennard-Jones (LJ) potential model

$$
\varphi(r)=\frac{\alpha}{r^{m}}-\frac{\beta}{r^{n}},
$$

where $m$ and $n$ depends on the interaction type, and their values will be discussed in Sec. IV. The force constant $K$ and the bond length $r_{0}$ at the equilibrium position are given as

$$
\begin{aligned}
& K=-\frac{m n \varphi_{o}}{r_{o}^{2}}=\frac{m(m-n) \alpha}{r_{o}^{(m+2)}}=n(m-n) \beta\left(\frac{\beta n}{\alpha m}\right)^{(n+2) /(m-n)}, \\
& r_{o}=\left(\frac{\alpha m}{\beta n}\right)^{1 /(m-n)},
\end{aligned}
$$

where $-\varphi_{o}$ is the potential energy at the equilibrium position. Equation (10) shows that the force constant at the equilibrium position is proportional to $\varphi_{o}$, when the bond type and the bond length are similar. Note that at the equilibrium position, the ratio of the magnitudes of the contributions from the repulsive term and the attractive term is $(m+1) /(n+1)$. Therefore, for $m \gg n$ (e.g., for ionic bond), the force constant is mainly determined by the repulsive term.

From Eqs. (7), (8), and (10), if $K_{A A}$ is defined as the force constant of the potential function $\varphi_{A A}(r)=\varphi_{A A, \text { rep }}(r)$ $-\varphi_{A A \text {,att }}(r)$, the force constant of $A-B$ bond $K_{A B}$ and its equilibrium bond length $r_{o, A B}$ can be given as

$$
K_{A B}=\left(K_{A A} K_{B B}\right)^{1 / 2}, r_{o, A B}=\left(r_{o, A A} r_{o, B B}\right)^{1 / 2} .
$$

Note that for ions, the $A-A$ bond may not actually exist. However, due to the similarity of the electronic configuration of the ions in different compounds, we may assign a virtual potential $\varphi_{A A}$ to the ions, e.g., keeping the interaction due to Pauli exclusion principle as the repulsive term and setting the attractive term as $\varphi_{A A \text {,att }}=q^{2} / r$, where $q$ is the ionic charge. The properties of the virtual potential (e.g., $K_{A A}$ ) can be extracted from the compounds. In this way, the combinative rule [Eqs. (7) and (8)] is still valid. Similar relations like Eq. (11) have been derived by Feranchuk et al. ${ }^{16}$ using 12-6 LJ potential, but they did not consider the effects of bond order and the long-range electrostatic interactions. In addition, it is not appropriate to describe ionic bonds or covalent bonds using 12-6 LJ potential, as will be discussed later.

Note that this combinative rule is only applicable for the bonds with the same bond type ( $m$ and $n$ are close) and bond order. In real compounds, a bond with the same atom configuration can have different bond orders. For example, C
$=\mathrm{O}$ has the bond order of 2, and $\mathrm{C}-\mathrm{O}$ has the bond order of 1. It is observed that the force constant is approximately proportional to the bond order, ${ }^{17}$ that is

$$
K_{A B, s}=s K_{A B, 1},
$$

where $K_{A B, s}$ is the force constant of the bond between $A$ and $B$ with the bond order of $s$. Thus Eq. (12) can be rewritten as

$$
K_{A B, s}=s\left(K_{A A, 1} K_{B B, 1}\right)^{1 / 2} .
$$

Consequently, we have

$$
K_{A C, s}=s \frac{\left(K_{A B, 1} K_{B C, 1}\right)^{1 / 2}}{K_{B B, 1}} .
$$

According to Eq. (12), the potential energy $\varphi$ can be assumed proportional to $s$, and $r_{e}$ is expected to be independent of $s$. For ionic bonds, when this assumption is used, the resulting combinative rule for ionic bond length agrees well with the experiments (the error is less than $3 \%$ ). ${ }^{16}$ However, this assumption is only moderately accurate for covalent bonds, because the LJ potential does not accurately describe the changes of electron clouds and the energy in the entire range of atomic distance. Generally, for covalent bonds, $r_{e}$ will decreases slightly when $s$ increases. Paolini ${ }^{18}$ developed an empirical bond order-bond length relationship for covalent bonds

$$
r_{e, s}=r_{e, 1}-0.78\left(s^{0.33}-1\right),
$$

where $r_{e, s}$ is the equilibrium bond length (in Angstrom) with the bond order of $s$. Equation (15) shows good agreements with the experimental results for many bonds ${ }^{18}$ and can be used for the estimation of the bond length.

Table I lists the force constant $K_{A A, 1}$, electronegativity $\chi$, and equilibrium bond length $r_{e, 1}$. $K_{A A, 1}$ is extracted from the experimental spectra of diatomic molecules ${ }^{19}$ according to Eqs. (13) and (14) [the ionic $K_{A A, 1}$ of elements, e.g., $\mathrm{O}$ and S, is an average of the values extracted from their compounds]. $r_{e, 1}$ is extracted from the bond lengths of the diatomic molecules. ${ }^{19}$ Table I shows that $K_{A A, 1}$ of ionic bonds for the elements with high electronegativity $\chi$ (e.g., $\mathrm{O}$ and $\mathrm{Cl}$ ) are normally twice that of the corresponding covalent bond. This indicates that the virtual potential of ions is steeper than the covalent potential of the corresponding atoms near the equilibrium position. The electronegativity $\chi$ can be used to determine the bond type. Bonds between atoms with a large electronegativity difference $(\geq 1.7)$, are usually considered to be ionic, while values between 1.7 and 0.4 are considered polar covalent, and values below 0.4 are considered nonpolar covalent bonds. ${ }^{20}$ For metallic elements, even though $\Delta \chi$ is small, their electron structures are similar to those in the ionic crystals, for the conduction electrons can move about. ${ }^{21}$

Figure 1(a) shows that generally the ionic $K_{A A, 1}$ increases as the electronegativity increases. The alkali metals have the lowest $K_{A A, 1}$, while the halogen elements have the highest $K_{A A, 1}$. When $1.0<\chi<2.5$, most transition metal elements and semiconducting elements have a $K_{A A, 1}$ around $50 \mathrm{~N} / \mathrm{m}$, which is a relatively low value. In general, $K_{A A, 1}$ 
TABLE I. Electronegativity, equilibrium bond length, and force constant of elements with the bond order of 1. The data are extracted from Ref. 19. The symbols $\mathrm{C}$ and $\mathrm{V}$ represent covalent and van der Waals interactions, and the unlabeled are the values for ionic interactions.

\begin{tabular}{|c|c|c|c|c|c|c|c|c|c|c|c|c|c|c|c|}
\hline Atom & $\chi$ & $\begin{array}{l}r_{e, 1} \\
(\AA)\end{array}$ & $\begin{array}{c}K \\
(\mathrm{~N} / \mathrm{m})\end{array}$ & Atom & $x$ & $\begin{array}{l}r_{e, 1} \\
(\AA)\end{array}$ & $\begin{array}{c}K \\
(\mathrm{~N} / \mathrm{m})\end{array}$ & Atom & $\chi$ & $\begin{array}{l}r_{e, 1} \\
(\AA)\end{array}$ & $\begin{array}{c}K \\
(\mathrm{~N} / \mathrm{m})\end{array}$ & Atom & $x$ & $\begin{array}{l}r_{e, 1} \\
(\AA)\end{array}$ & $\begin{array}{c}K \\
(\mathrm{~N} / \mathrm{m})\end{array}$ \\
\hline $\mathrm{H}$ & 2.20 & $0.74[\mathrm{C}]$ & $575.67[\mathrm{C}]$ & $\mathrm{Ar}$ & - & $3.76[\mathrm{~V}]$ & $0.80[\mathrm{~V}]$ & $\mathrm{Br}$ & 2.96 & $2.28[\mathrm{C}]$ & $\begin{array}{l}250.83[\mathrm{C}], \\
539.78\end{array}$ & $\mathrm{La}$ & 1.10 & 2.83 & 53.41 \\
\hline $\mathrm{He}$ & - & $1.04[\mathrm{C}]$ & $411.74[\mathrm{C}]$ & K & 0.82 & 3.90 & 9.84 & $\mathrm{Kr}$ & 3.00 & $4.03[\mathrm{~V}]$ & $1.43[\mathrm{~V}]$ & $\mathrm{Ce}$ & 1.12 & 2.74 & 169.30 \\
\hline $\mathrm{Li}$ & 0.98 & 2.67 & 25.48 & $\mathrm{Ca}$ & 1.00 & 4.28 & 34.61 & $\mathrm{Rb}$ & 0.82 & 3.79 & 8.25 & $\operatorname{Pr}$ & 1.13 & - & 48.25 \\
\hline $\mathrm{Be}$ & 1.57 & 1.39 & 120.62 & $\mathrm{Sc}$ & 1.36 & 2.40 & 77.60 & $\mathrm{Sr}$ & 0.95 & 3.05 & 26.57 & $\mathrm{Eu}$ & 1.20 & - & 31.11 \\
\hline B & 2.04 & $1.76[\mathrm{C}]$ & $354.90[\mathrm{C}]$ & $\mathrm{Ti}$ & 1.54 & 2.17 & 107.71 & $\mathrm{Y}$ & 1.22 & - & 70.05 & $\mathrm{~Tb}$ & 1.10 & - & 72.63 \\
\hline $\mathrm{C}$ & 2.55 & $1.54[\mathrm{C}]$ & $610.5[\mathrm{C}]$ & $\mathrm{V}$ & 1.63 & 2.09 & 103.03 & $\mathrm{Zr}$ & 1.33 & 8.41 & 141.43 & Ho & 1.23 & 3.11 & 77.21 \\
\hline $\mathrm{N}$ & 3.04 & $1.46[\mathrm{C}]$ & $771.20[\mathrm{C}]$ & $\mathrm{Cr}$ & 1.66 & 2.17 & 87.18 & $\mathrm{Nb}$ & 1.60 & 2.36 & 108.25 & $\mathrm{Yb}$ & 1.10 & 2.89 & 33.74 \\
\hline $\mathrm{O}$ & 3.44 & $1.46[\mathrm{C}]$ & $\begin{array}{l}593.57[\mathrm{C}] \\
1305\end{array}$ & $\mathrm{Mn}$ & 1.55 & 2.59 & 46.40 & $\mathrm{Ru}$ & 1.02 & 2.17 & 56.53 & $\mathrm{Lu}$ & 1.27 & 2.63 & 78.69 \\
\hline $\mathrm{F}$ & 3.98 & $1.41[\mathrm{C}]$ & $\begin{array}{l}473.82[\mathrm{C}] \\
1960\end{array}$ & $\mathrm{Fe}$ & 1.83 & 2.04 & 62.53 & $\mathrm{Ag}$ & 1.93 & 2.59 & 59.09 & $\mathrm{Hf}$ & 1.30 & 2.44 & 103.98 \\
\hline $\mathrm{Ne}$ & - & $3.10[\mathrm{~V}]$ & $0.12[\mathrm{~V}]$ & Co & 1.88 & 3.20 & 116.61 & $\mathrm{Cd}$ & 1.69 & 4.28 & 44.93 & $\mathrm{Ta}$ & 1.50 & 2.36 & 179.07 \\
\hline $\mathrm{Na}$ & 0.93 & 3.08 & 17.28 & $\mathrm{Ni}$ & 1.91 & 2.96 & 130.00 & In & 1.78 & 2.86 & 34.41 & $\mathrm{~W}$ & 2.36 & - & 202.54 \\
\hline $\mathrm{Mg}$ & 1.31 & 3.89 & 41.60 & $\mathrm{Cu}$ & 1.90 & 2.22 & 65.82 & Sn & 1.96 & 2.80 & 58.34 & Ir & 2.20 & 2.36 & 110.01 \\
\hline $\mathrm{Al}$ & 1.61 & 2.47 & 49.15 & $\mathrm{Zn}$ & 1.65 & 3.41 & 81.15 & $\mathrm{Sb}$ & 2.05 & $2.82[\mathrm{C}]$ & $70.64[\mathrm{C}]$ & $\mathrm{Au}$ & 2.54 & 2.47 & 106.80 \\
\hline $\mathrm{Si}$ & 1.90 & $2.34[\mathrm{C}]$ & $109.04[\mathrm{C}]$ & $\mathrm{Ga}$ & 1.81 & 2.43 & 99.38 & $\mathrm{Te}$ & 2.10 & $2.74[\mathrm{C}]$ & $119.46[\mathrm{C}]$ & $\mathrm{Hg}$ & 2.00 & 3.30 & 32.61 \\
\hline$P$ & 2.19 & $2.20[\mathrm{C}]$ & $201.50[\mathrm{C}]$ & $\mathrm{Ge}$ & 2.01 & 2.16 & 121.79 & I & 2.66 & $2.66[\mathrm{C}]$ & $172.73[\mathrm{C}] 343.06$ & $\mathrm{Tl}$ & 1.62 & 3.07 & 31.60 \\
\hline S & 2.58 & $2.08[\mathrm{C}]$ & $\begin{array}{l}250.65[\mathrm{C}] \\
536.92\end{array}$ & As & 2.18 & 2.42 & 120.48 & $\mathrm{Xe}$ & 2.60 & $4.36[\mathrm{~V}]$ & $1.74[\mathrm{~V}]$ & $\mathrm{Pb}$ & 2.33 & 3.03 & 39.91 \\
\hline $\mathrm{Cl}$ & 3.16 & $1.98[\mathrm{C}]$ & $\begin{array}{l}330.42[\mathrm{C}] \\
705.81\end{array}$ & $\mathrm{Se}$ & 2.55 & $2.34[\mathrm{C}]$ & $108.54[\mathrm{C}]$ & Cs & 0.79 & 4.47 & 6.97 & $\mathrm{Bi}$ & 2.02 & 3.07 & 49.08 \\
\hline
\end{tabular}

decreases while the atomic radius increases. However, Fig. 1(b) shows for covalent bonds $K_{A A, 1}$ seems to relate to the ratio of $\chi / N(N$ is the atomic number) rather than $\chi$. Nitrogen has the highest covalent $K_{A A, 1}$.

Figure 2 compares the experimental results of some bonds in diatomic molecules along with the calculated values. The mean square error is less than $8 \%$, and the overall agreement is good.

Note that the above force constants and equilibrium bond lengths are derived from the data of gaseous diatomic molecules, where the intermolecular effects are negligible. For crystal bonds, long-range interactions (mainly electrostatic interactions) from the surroundings may significantly affect the equilibrium bond length and force constant. For example, $\mathrm{Na}-\mathrm{Cl}$ in a $\mathrm{NaCl}$ molecule has a force constant of $110 \mathrm{~N} / \mathrm{m}$ and a bond length $2.36 \AA{ }^{19}$ while the distance between the nearest $\mathrm{Na}$ and $\mathrm{Cl}$ ions in a $\mathrm{NaCl}$ crystal at $T$ $=300 \mathrm{~K}$ is $2.83 \AA$, and the effective force constant of each $\mathrm{Na}-\mathrm{Cl}$ pair derived from the bulk modulus is only $20 \mathrm{~N} / \mathrm{m} .^{22}$ Thus, a relation between the force constant of a bond in a gaseous diatomic molecule and that in a crystal must be developed to account for the effects of long-range interactions. Here, only the effect of electrostatic interactions is considered. Our approach is to include long-range interactions in an effective bond potential of the nearest neighboring atoms. A bond (in a diatomic molecule) with a form in Eq. (9) is considered. Since the repulsive term is a very short-range interaction, we assume only the long-range attractive term is affected by the surroundings. This effective bond in a crystal can then be represented as

$$
\langle\varphi\rangle(r)=\frac{\alpha}{r^{m}}-\eta \frac{\beta}{r^{n}},
$$

where $\eta$ is the correction factor due to the long-range interactions (in simple ionic structures, it is related to the Madelung constant). However, $\eta$ is difficult to determine for complex crystal structures. In practice, according to this assumption and Eq. (10), the force constant of the bond in the crystal $K_{A B}^{\prime}$ can be simply calculated as

$$
K_{A B}^{\prime}=K_{A B}\left(\frac{r_{o}}{r_{o}^{\prime}}\right)^{(m+2)},
$$

where $K_{A B}$ is the force constant of the bond in the diatomic molecule $A B$, and $r_{o}^{\prime}$ is the equilibrium bond length in the crystal. For example, for $\mathrm{NaCl}$, by setting $m=6.3$ [using the approximation method Eq. (36)], and using the above bond length data, we have $K_{\mathrm{NaCl}}^{\prime}=110 \times(2.36 / 2.83)^{(6.3+2)}$ $=24 \mathrm{~N} / \mathrm{m}$, which is very close to $20 \mathrm{~N} / \mathrm{m}$ derived from the bulk modulus. $^{22}$ For ionic bonds, $m$ is large, and Eq. (17) indicates that $K_{A B}^{\prime}$ is very sensitive to the values of $r_{o}$ and $r_{o}^{\prime}$, so the experimental values of $r_{o}$ and $r_{o}^{\prime}$ will be preferred. When the experimental value of $r_{o}$ is unavailable, the combinative rule [Eq. (11)] can be used.

\section{EVALUATION OF SOUND VELOCITY AND DEBYE TEMPERATURE}

If the force constants between atoms are known, the dynamical matrix can be readily constructed to determine the sound velocity. However, for complex polyatomic crystals, the calculation is still very cumbersome, and it is difficult to explicitly relate the numerical results to the complex structure. For the purpose of estimation and design, a simple 


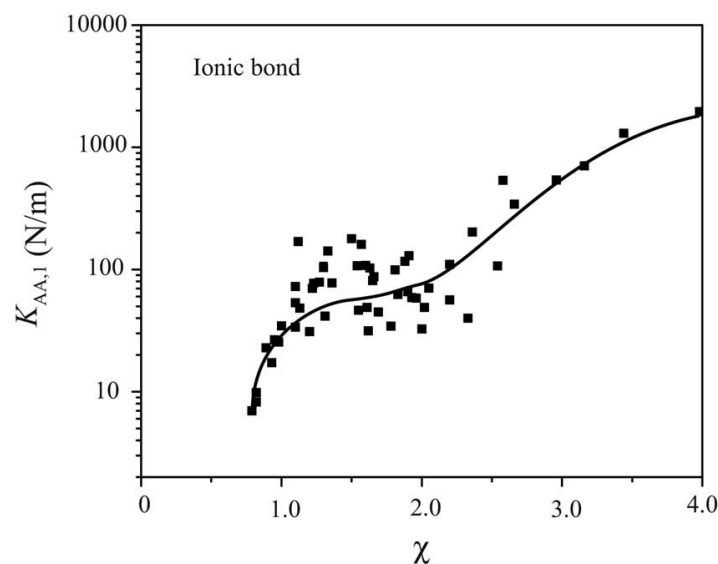

(a)

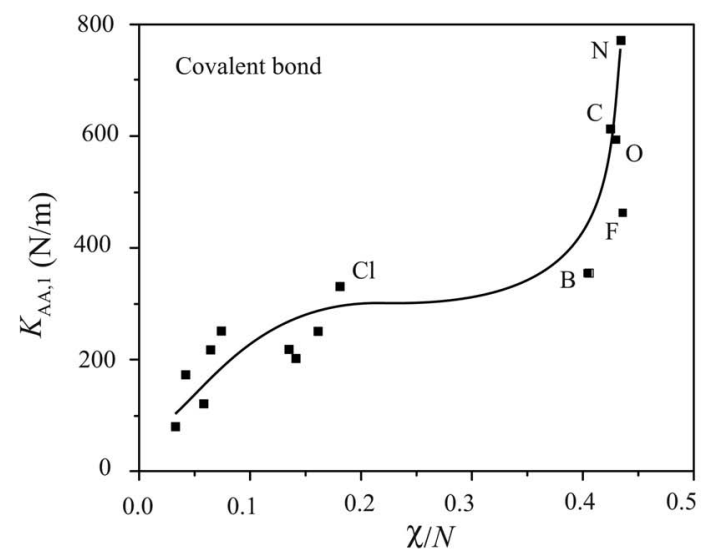

(b)

FIG. 1. (a) Variation of calculated ionic force constant $K_{A A, 1}$ with respect to the electronegativity. (b) Variation of the calculated covalent force constant $K_{A A, 1}$ with respect to the ratio of the electronegativity and atomic number. The data are extracted from the spectra of diatomic molecules (Ref. 19). The lines are used to guide the eyes.

model that can directly relate the sound velocity and the Debye temperature to the crystal structure is needed.

A real crystal structure can always be considered as an underlying lattice, together with a basis describing the arrangement of the atoms, ions, and molecules within a primitive cell. ${ }^{13}$ The acoustic branches of the phonon dispersion

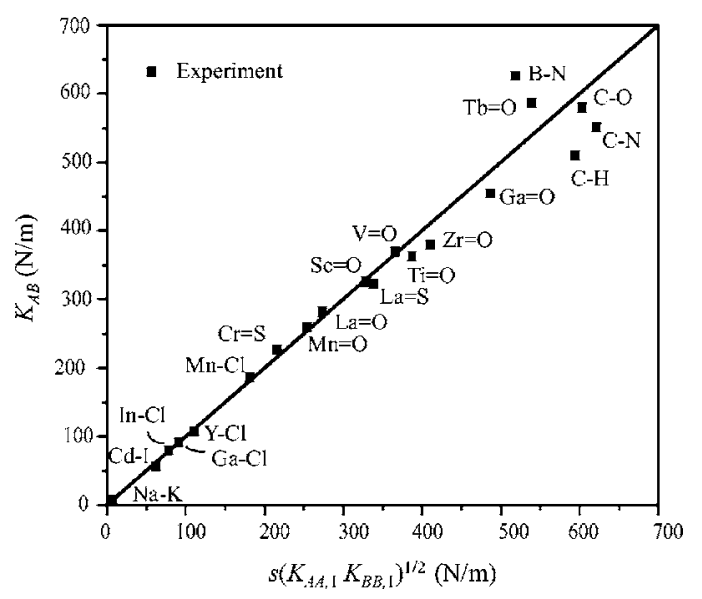

FIG. 2. Comparison of calculated force constants and the corresponding values from the experimental spectra, for some atomic pairs (Ref. 19).

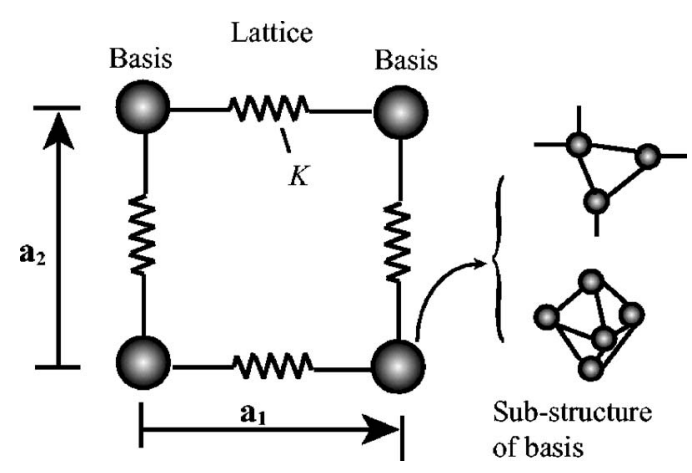

FIG. 3. Decomposition of a complex crystal into lattice and bases, with equivalent bonds.

correspond to the motion of the mass centers of the primitive cells. ${ }^{13}$ Therefore, both the monatomic and polyatomic crystal structures can be modeled as a lattice with rigid bases connected by equivalent bonds with an equivalent force constant, as shown in Fig. 3.

\section{A. Equivalent force constants}

The elastic response of a solid can be divided into two parts, namely, (i) atomic vibrations at fixed volume, and (ii) unit cell volume fluctuations for a fixed atomic configuration (homogeneous deformation). The first part corresponds to the inhomogeneous deformation, in which the bending potentials and the torsion potentials may be important, especially in a flexible structure. The bending potential can also be converted into an equivalent stretching potential between the atoms at the two ends. Since the force constant of the bending potentials and the torsion potentials are normally small, the equivalent force constant will be much reduced by the inhomogeneous deformation. It is difficult to obtain a general simple analytical solution for such an inhomogeneous deformation, and a numerical calculation using full dynamical matrix (including the bending potentials) is preferred for obtaining the equivalent force constant. However, for many solids, the crystallographic symmetries and the stability of a given phase with respect to small lattice deformations result in the diminishing effects from the first part, ${ }^{23}$ and the elastic behavior can be described using the equivalent force constants of the stretching potentials. In these cases, the bending potential and the torsion potential may contribute to the stability of the structure, but their contribution to the elastic response is negligible.

When only considering the bond stretching, it is apparent that only the transport of stretching along the translational unit vector a can contribute to the energy transport in this direction. Thus we define the force constant of a bond along a given unit vector $\mathbf{a}$ as $^{24}$

$$
K_{\mu \nu, \mathbf{a}}=\frac{\partial^{2} \varphi_{\mu \nu}}{\partial x_{\mathbf{a}}^{2}}=\frac{\partial^{2} \varphi_{\mu \nu}}{\partial r_{\mu \nu}^{2}}\left(\frac{x_{\mathbf{a}}}{r_{\mu \nu}}\right)^{2}=\left(\mathbf{a} \cdot \mathbf{n}_{\mu \nu}\right)^{2} K_{\mu \nu},
$$

where $x_{\mathbf{a}}$ is the projection of the bond length $r$ along $\mathbf{a}$, and $\mathbf{n}_{\mu \nu}$ is the unit vector pointing from the particle $\mu$ to the particle $\nu$. Equation (18) shows that the projection of the force constant along $\mathbf{a}$ has a factor of $\left(\mathbf{a} \cdot \mathbf{n}_{\mu \nu}\right)^{2}$. 
The total deformation of the primitive cell is affected by all the bonds in it. Using Eq. (18), we may treat the bonds in a primitive cell as springs with the same $K_{\mu \nu, \mathbf{a}}$, and then convert the crystal primitive cell into a network composed of springs. This spring network can be simplified to obtain the equivalent force constant between two bases according to the following rules (we denote the force constants of two bonds as $K_{1}$ and $K_{2}$, and that of the equivalent bond of these two bonds as $K_{\mathrm{eq}}$ ):

(i) When the two bonds are in series,

$$
K_{\text {eq }}^{-1}=K_{1}^{-1}+K_{2}^{-1} \text {; }
$$

(ii) when the two bonds are parallel,

$$
K_{\text {eq }}=K_{1}+K_{2} \text {. }
$$

For a monatomic crystal, the primitive cell only includes one atom, and the equivalent force constant is just the force constant of the bond between the atoms.

\section{B. Sound velocity and Debye temperature model}

From the lattice dynamics, the sound velocities of acoustic branches at the long-wavelength limit are the square roots of the eigenvalues of the matrix ${ }^{13}$

$$
-\frac{1}{2 M} \sum_{\mathbf{R}}\left(\mathbf{s}_{\kappa} \cdot \mathbf{R}\right)^{2} \mathbf{D}(\mathbf{R}), D_{i, j}(\mathbf{R})=\frac{\partial^{2} \varphi}{\partial u_{i}^{0} \partial u_{j}^{\mathbf{R}}},
$$

where $\mathbf{s}_{\kappa}$ is the unit wave vector, $\mathbf{D}(\mathbf{R})$ is the force matrix, $\mathbf{R}$ is the position vector of the neighbor, $u$ is the displacement of the mass center of the primitive cell from the equilibrium position ( 0 represents the origin), and $M$ is the mass of the primitive cell.

Using the above simplified model for crystal structures, if only the stretching energy is considered, Eq. (5) can be rewritten as

$$
\phi=\sum_{\mathbf{R}} \frac{K_{\mathbf{R}}}{2}\left\{\mathbf{n}_{\mathbf{R}} \cdot\left[\mathbf{u}^{\mathbf{R}}-\mathbf{u}^{0}\right]\right\}^{2}, \quad \mathbf{n}_{\mathbf{R}}=\frac{\mathbf{R}}{|\mathbf{R}|},
$$

where $K_{R}$ is the equivalent force constant between the two bases. Thus $D_{i, j}(\mathbf{R})=\eta_{i, j} K_{\mathbf{R}}$. Note that $\mathbf{R}$ is a linear function of the lattice constants, so the sound velocity will have the form

$$
u_{p, g, i}=d\left(\mathbf{s}_{k, i},\left\{\mathbf{a}_{i}\right\}\right)\left(\frac{\Gamma_{i}}{M}\right)^{1 / 2}, \Gamma_{i}=\sum_{\mathbf{R}}\left[\eta\left(\mathbf{R}, \mathbf{s}_{k, i}\right) K_{\mathbf{R}}\right],
$$

where $\left\{\mathbf{a}_{i}\right\}$ is the set of the translational vectors of the lattice. Note that Eq. (23) has the same form as the formula for the one-dimensional chain. ${ }^{24}$ It is instructive to consider a plane wave traveling in a crystal, wherein the lattice is consisted of parallel planes perpendicular to the wave vector and the atoms in a plane will move in phase. The transportation along the wave vector is essentially one dimensional. From the comparison with the formula of the one-dimensional chain, ${ }^{24}$ $d$ is indeed the equivalent distance between the planes and normally is the linear function of the lattice constants. The effective force constant $\Gamma$ is the summation of the projections of the equivalent force constant in the polarization $\mathbf{s}_{\kappa, i}$, that is, $\eta\left(\mathbf{R}, \mathbf{s}_{\kappa, i}\right)=\left(\mathbf{n}_{\mathbf{R}} \cdot \mathbf{s}_{\kappa, i}\right)^{2}$.

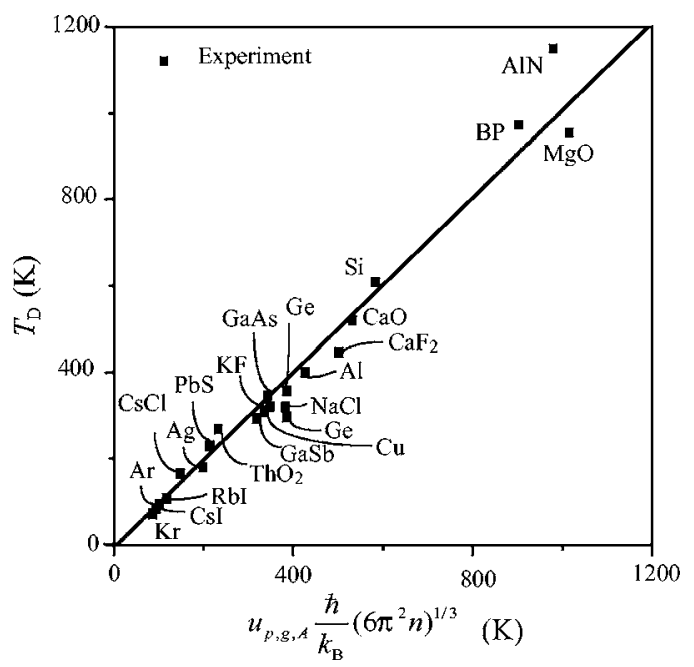

FIG. 4. Comparison of the predicted and measured Debye temperature for some crystals. The force constants for metallic crystals are calculated according to Eq. (17) ( $m$ is set to 8 ). The experimental values are from Ref. 11.

The average sound velocity $u_{p, g, \mathrm{~A}}$ can be calculated as

$$
u_{p, g, \mathrm{~A}}=\left(\sum_{i=1}^{3} \frac{1}{3 u_{p, g, i}^{3}}\right)^{-1 / 3} .
$$

For cubic structures, the average sound velocity can be given as

$$
u_{p, g, \mathrm{~A}}=\frac{1}{3^{1 / 2}} a\left(\frac{K}{M}\right)^{1 / 2}
$$

where $a$ is the lattice constant.

From the longitudinal and transversal sound velocity, we can obtain the polarization-dependent Debye temperature $T_{D, i}$ and the average Debye temperature $T_{D}{ }^{13}$

$$
\begin{aligned}
& T_{D, i}=u_{p, g, i} \frac{\hbar}{k_{B}}\left(6 \pi^{2} n_{a}\right)^{1 / 3}=\frac{d_{i}}{V_{c}^{1 / 3}}\left(\frac{\Gamma_{i}}{M}\right)^{1 / 2} \frac{\hbar}{k_{B}}\left(6 \pi^{2} N_{c}\right)^{1 / 3}, \\
& T_{D}=u_{p, g, A} \frac{\hbar}{k_{B}}\left(6 \pi^{2} n_{a}\right)^{1 / 3}=\left(\sum_{i=1}^{3} \frac{1}{3 T_{D, i}^{3}}\right)^{-1 / 3},
\end{aligned}
$$

where $n_{a}$ is the number density of atoms, $N_{c}$ is the number of atoms in a primitive cell, and $V_{c}$ is the volume of a primitive cell. Here $d_{i} / V_{c}^{1 / 3}$ is only a function of the ratio of lattice constants and the polarization, and the Debye temperature relates to the ratio of the lattice constants rather than their absolute values. It is apparent that if the lattice constant and other parameters are the same except the lattice type, the order of magnitude of $T_{D}$ is $T_{D}(\mathrm{FCC})>T_{D}(\mathrm{BCC})>T_{D}(\mathrm{SC})$.

Figure 4 compares the calculated and experimental Debye temperatures ${ }^{11}$ (determined from elastic constants or specific heat capacity measurements) of some crystals. The force constants used in the calculation are from the combinative rule and Table I. The overall agreement is good. The force constant for metals are calculated according to Eq. (17). It is found that $m=8$ gives good agreement with experimental values. Again, it is found that for metallic crystals, 
the force constant can be reduced significantly by the longrange electrostatic interaction (by a factor of about 5), which results in a low Debye temperature.

\section{THERMAL EXPANSION AND GRÜNEISEN PARAMETER}

The Grüneisen parameter has been used to represent the volume dependence of the normal mode frequencies. The overall Grüneisen parameter $\gamma$ is defined as ${ }^{13}$

$$
\gamma=\frac{\sum_{\kappa, \alpha} \gamma_{\kappa, \alpha} c_{v \alpha}(\kappa)}{\sum_{\kappa, \alpha} c_{v \alpha}(\kappa)}, \quad \gamma_{\kappa, \alpha}=-\frac{\partial \ln \omega_{\kappa, \alpha}}{\partial \ln V},
$$

where the subscript $\alpha$ denotes the branch of a normal mode, $c_{v}$ is the heat capacity per normal mode, and $V$ is the volume. In the Debye approximation, all the normal-mode frequencies scale linearly with the Debye temperature $T_{D}$ and, therefore, ${ }^{13}$

$$
\gamma=\gamma_{\kappa, \alpha}=-\frac{\partial \ln \omega_{D}}{\partial \ln V} .
$$

That is, $\gamma$ represents the relative shift of the Debye angular frequency with respect to the volume.

We consider a crystal containing only one bond type. According to Eqs. (23) and (28), since the Debye frequency $\omega_{D} \propto K^{1 / 2}$ and the volume $V \propto r_{e}^{3}$, we have

$$
\gamma=-\frac{\mathrm{d} \ln K}{6 \mathrm{~d} \ln r_{e}} .
$$

Note that $\gamma$ only relates to the bond. Zallen ${ }^{25}$ defined a "bonding-scaling parameter" $\gamma^{\prime}$ as

$$
\gamma_{i}^{\prime}=-\frac{\mathrm{d} \ln K_{i}}{6 \mathrm{~d} \ln r_{i}^{\prime}},
$$

where $K_{i}^{\prime}$ and $r_{i}^{\prime}$ are the force constant and the equilibrium length of the bond $i$. For the crystals containing only one bond type, the Grüneisen parameter $\gamma$ is equal to the bonding-scaling parameter $\gamma^{\prime}$.

We can rewrite Eq. (30) in terms of a small relative deviation fraction $\varepsilon$ :

$$
K_{i}^{\prime}(\varepsilon) \simeq K_{o}^{\prime}\left(1-6 \gamma^{\prime} \varepsilon\right),
$$

where $K_{i}^{\prime}$ is the initial equivalent force constant. It is apparent that $\gamma^{\prime}$ represents the intrinsic anharmonicity of a bond, i.e., the relative shift of the force constant with respect to the bond length. It seems reasonable that the $\gamma^{\prime}$ of each bond is independent of other bonds.

We again consider the crystal containing one bond type to obtain the bond-scaling parameter $\gamma^{\prime}$. Ruffa ${ }^{26}$ developed a thermodynamic description of Morse oscillators using a statistical treatment. Here a similar approach is applied for the Lennard-Jones oscillators representing the interatomic potentials. Consider an assembly of independent oscillators with the interatomic potential of Eq. (9), of which the natural angular frequency $\omega=(K / \mu)^{1 / 2}$ ( $\mu$ is the reduced mass of the oscillator). If Eq. (9) is expanded in a Taylor series, the vibrational energy $E_{l}$ and the mean atomic separation $\left\langle r_{l}\right\rangle$ of the motion with the principle quantum number $l$ of this oscillator can be expressed as ${ }^{27}$

$$
\begin{aligned}
& E_{l}=\hbar \omega\left(l+\frac{1}{2}\right)-C_{e} \hbar^{2} \omega^{2}\left(l+\frac{1}{2}\right)^{2}, C_{e}=\frac{5(m+n+3)^{2}}{48 \varphi_{o} m n}, \\
& \left\langle r_{l}\right\rangle=r_{o}+C_{r} r_{o} \hbar \omega\left(l+\frac{1}{2}\right), C_{r}=\frac{3(m+n+3)}{2 \varphi_{o} m n}
\end{aligned}
$$

Then, we have (see the derivation in the Appendix)

$$
\begin{aligned}
& \gamma^{\prime} \simeq \gamma_{o}^{\prime}\left[1+C_{e} k_{B} T \frac{f_{1}\left(x_{D}\right)}{f\left(x_{D}\right)}\right], \quad \gamma_{o}^{\prime}=\frac{m+n+3}{6}, \\
& f\left(x_{D}\right)=\int_{0}^{x_{D}} \frac{x^{3} \mathrm{~d} x}{e^{x}-1}, x_{D}=T_{D} / T, \\
& f_{1}\left(x_{D}\right)=\int_{0}^{x_{D}} \frac{x^{4}\left(1+e^{x}\right) \mathrm{d} x}{\left(e^{x}-1\right)^{2}} .
\end{aligned}
$$

Typically, the vibration energy is much smaller than the dissociation energy and $C_{e} k_{B} T$ is small, so the temperature dependence of $\gamma^{\prime}$ is weak. At high temperatures, $\gamma^{\prime}$ will reach $\gamma_{o}^{\prime}$. It is very interesting to note that $\gamma_{o}^{\prime}$ only depends on $m$ and $n$, or the bond type.

(i) Ideal ionic bonds. The attractive potential is dominated by the electrostatic potential, and the lattice summation of the long-range electrostatic interactions does not change $n$ (the Madelung term), thus $n=1$. The repulsive term arises from the full-filled shells and the Pauli exclusion principle. The measurements for typical ionic bonds show $m=6-10 .^{13}$ The midpoint $m=8$ is a reasonable choice for the estimation, therefore, $\gamma_{o}^{\prime}=2.0$. In fact, $\gamma_{o}^{\prime}=2.0$ agrees well with the high temperature $\gamma$ values of many typical ionic crystals with one bond type. 11,24

(ii) van der Waals interactions. The attractive term arises from the interaction between dipoles and varies as $1 / r^{6}$, that is, $n=6$. The widely-used 12-6 Lennard-Jones potential chooses $m=12$ for the repulsive term. However, it is found that $m=12$ makes the repulsive term very steep. ${ }^{5} m=12$ gives $\gamma_{o}^{\prime}=3.5$, a much higher value than the measured results. For example, at high temperatures, $\gamma$ of $\mathrm{Ne}, \mathrm{Ar}, \mathrm{Kr}$, and $\mathrm{Xe}$ are $2.76,2.73,2.84,2.65,{ }^{11}$ respectively. Considering the repulsive term of van der Waals interaction arises from the same mechanism (i.e., the filled outer shell) as in an ionic bond, it is reasonable to choose the same value 8 for $m$. This choice gives $\gamma_{o}^{\prime}=2.83$, which agrees much better with the above experimental results.

(iii) Nonpolarized covalent bonds. The attractive term is due to the electrostatic interaction, therefore, $n=1$. For $m$, the case is more complicated, because the distribution of valence electrons differs substantially from that in isolated atoms or ions. The repulsive term includes the electrostatic term and the term due to the Pauli exclusion principle. In fact, the covalent bond is more appropriately described by the Morse potential $^{5}$

$$
\varphi=\varphi_{o}\left[e^{-2 a_{o}\left(r-r_{o}\right)}-2 e^{-a_{o}\left(r-r_{o}\right)}\right] .
$$

Ruffa $^{26}$ suggested an empirical relation: $a_{o} r_{o}=(m+4) / 5$. For typical covalent bonds, $a_{o} r_{o} \simeq 1.0-1.2$, thus $m \simeq 1-2$. Since $m>n$, we choose $m=2$ and obtain $\gamma_{o}^{\prime}=1.0$. This value is also 
in accord with the relation $\mathrm{Kr}^{6}=$ const for covalent bonds, as suggested by Herzberg. ${ }^{14}$

The covalent bond between atoms with different electronegativities is partially polarized (ionic bonds can also be considered highly polarized covalent bonds). Using the relation of the percent of the ionic character $c$ proposed by Pauling, ${ }^{20} \gamma^{\prime}$ of a polarized bond can be given as

$$
\gamma^{\prime}=\gamma_{A B}^{\prime}=(1-c) \gamma_{\mathrm{cov}}^{\prime}+c \gamma_{\mathrm{ion}}^{\prime}, \quad c=1-e^{-\left(\chi_{A}-\chi_{B}\right)^{2} / 4},
$$

where $\gamma_{\text {cov }}^{\prime}$ and $\gamma_{\text {ion }}^{\prime}$ represent the bond-scaling parameters of a non-polarized covalent bond and the ideal ionic bond, respectively. Equation (35) together with Eq. (33) can also be used for the rough estimation of $m$ in an interatomic potential:

$$
m \simeq 8-6 e^{-\left(\chi_{A}-\chi_{B}\right)^{2} / 4} .
$$

(iv) Metallic bonds. Though metallic crystals also include ions, they are very different from ionic crystals. The metals can be treated as ions immersed in a sea of free electrons. ${ }^{13}$ Thus, the interactions between ions can be treated as the summation of the bare interactions between ions and the electron-ion interactions. Both the repulsive term and attractive term include the long-range electrostatic interactions. However, due to the screening effects of free electrons, the interaction between ions decays faster than the pure coulomb interactions, thus, $m>1$ and $n \geq 1$ (due to the attractions of ions to free electrons, repulsive term decays faster than the attractive term). The derivation of $\gamma^{\prime}$ for metallic bonds is complicated. To compare it with experimental results, one also needs to include the contribution from the free electrons (it may be small at high temperatures). However, since the screening effects increase with the increasing electron number density, ${ }^{13}$ we would expect that in the metals with high electron number density, $m \simeq 8$ and $1 \leq n \leq 6$. For simplicity, in this work we set $m=8$ and $n=1$ (the same values for ionic bonds). The resulting $\gamma=2.0$ is close to the experimental results of many metals (the alkali metals have a $\gamma$ close to 1.2 , due to the poor screening effects).

(v) Other interactions. Some other interactions, e.g., iondipole interaction, may exist in some crystals. These interaction may be considered as the cross terms of the above interactions. Using the combinative rule for potentials [Eqs. (8) and (7)], we can have

$$
m=\frac{\left(m_{1}+m_{2}\right)}{2}, n=\frac{\left(n_{1}+n_{2}\right)}{2}, \gamma^{\prime}=\frac{\left(\gamma_{1}^{\prime}+\gamma_{2}^{\prime}\right)}{2},
$$

where the subscripts 1 and 2 denote the individual interactions.

It can be seen that the order of magnitude of $\gamma^{\prime}$ for bonds is $\gamma^{\prime}$ (van der Waals bond) $>\gamma^{\prime}$ (ionic bond) $>\gamma^{\prime}$ (polarized covalent bond) $>\gamma^{\prime}$ (nonpolarized covalent bond). Figure 5 compares the calculated high-temperature Grüneisen parameters of crystals containing only one bond type with the experimental results (at the Debye temperature), ${ }^{11}$ and the overall agreement is good. Note that for ionic crystals, Grüneisen parameters are slightly overestimated. One reason is that the temperature at which the measurements are performed is not high enough. For example, $\gamma$

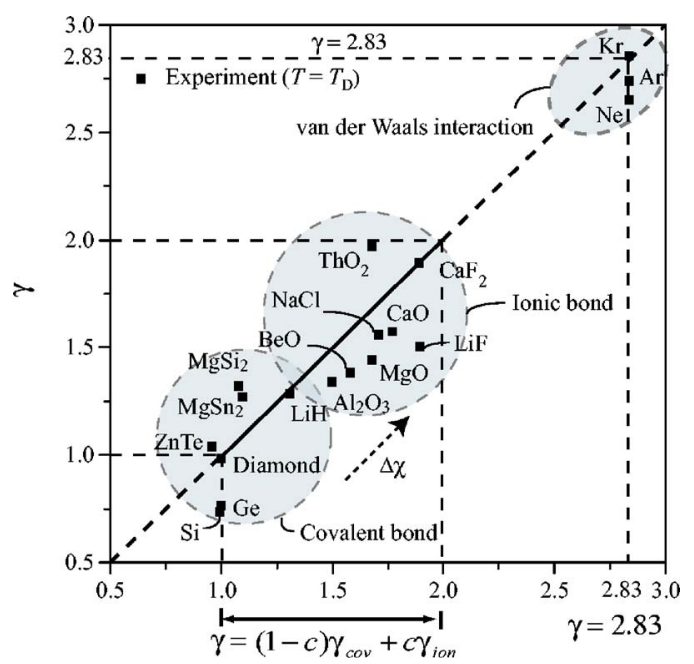

FIG. 5. Comparison of predicted high temperature Grüneisen parameters with the experimental results at the Debye temperatures, for some crystals (Ref. 11).

of $\mathrm{NaCl}$ at the Debye temperature is 1.57 , but at $800 \mathrm{~K}$, its value is $1.76,{ }^{28}$ compared to 1.71 given by Eq. (35). Another possible reason is that $c$ in Eq. (35) determined by Pauling is not very accurate.

Note that Eq. (30) is valid for the equivalent force constant $K$, so the Grüneisen parameter $\gamma$ of a crystal can be obtained by evaluating the equivalent $\gamma^{\prime}$ of the equivalent bond.

For two parallel bonds, Eqs. (20) and (31) lead to

$$
\gamma^{\prime}=\frac{K_{10}}{K_{10}+K_{20}} \gamma_{1}^{\prime}+\frac{K_{20}}{K_{10}+K_{20}} \gamma_{2}^{\prime},
$$

where $K_{10}$ and $K_{20}$ represent the equilibrium force constants of bond 1 and 2. That is, the equivalent $\gamma^{\prime}$ of the parallel bonds is the summation of the $\gamma_{\mathrm{i}}^{\prime}$ of the bonds weighted by the fraction of force constants.

Similarly, for two bonds in series, the equilibrium requirement gives

$$
\varepsilon_{1}=\frac{K_{20}\left(r_{1}+r_{2}\right)}{\left(K_{10}+K_{20}\right) r_{1}} \varepsilon, \quad \varepsilon_{2}=\frac{K_{10}\left(r_{1}+r_{2}\right)}{\left(K_{10}+K_{20}\right) r_{2}} \varepsilon,
$$

and Eqs. (19) and (31) lead to

$$
\gamma^{\prime}=\left(\frac{K_{20}}{K_{10}+K_{20}}\right)^{2} \frac{r_{1}+r_{2}}{r_{1}} \gamma_{1}^{\prime}+\left(\frac{K_{10}}{K_{20}+K_{10}}\right)^{2} \frac{r_{1}+r_{2}}{r_{2}} \gamma_{2}^{\prime} .
$$

Equation (40) shows the equivalent $\gamma^{\prime}$ is related not only to the force constants and $\gamma_{i}^{\prime}$, but also to the bond lengths. Note when $\varepsilon_{1}=\varepsilon_{2}=\varepsilon$ (homogeneous deformation), Eq. (40) can be reduced to

$$
\gamma^{\prime}=\left(\frac{K_{20}}{K_{10}+K_{20}}\right) \gamma_{1}^{\prime}+\left(\frac{K_{10}}{K_{20}+K_{10}}\right) \gamma_{2}^{\prime},
$$

which does not relate to the bond lengths.

Assuming $r_{1} \simeq r_{2}$ and $\gamma_{2}^{\prime}$ is the smaller one, the dependence of $\gamma^{\prime} / \gamma_{2}^{\prime}$ on the ratio of force constants $K_{10} / K_{20}$ is plotted in Fig. 6. 


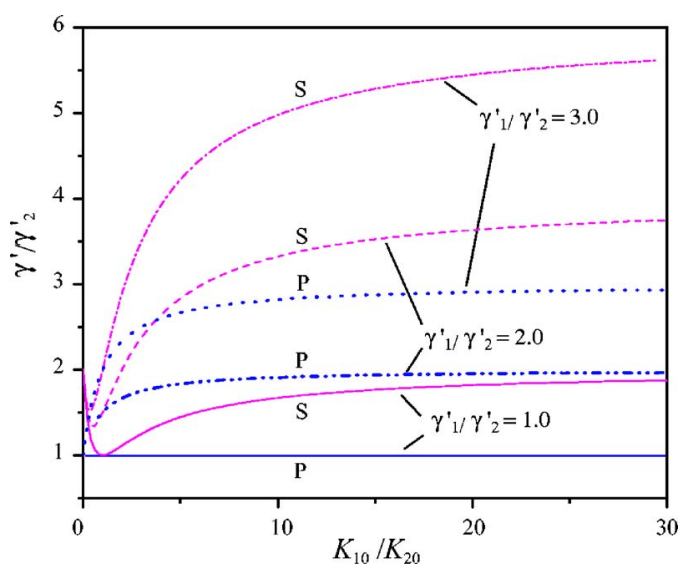

FIG. 6. (Color online) Variation of ratio of the equivalent bond-scaling parameter to the smaller bond-scaling parameter of the bonds $\gamma^{\prime} / \gamma_{2}^{\prime}$ with respect to the ratio of the force constants $K_{10} / K_{20}$. The symbols $\mathrm{P}$ and $\mathrm{S}$ denote the parallel and serial arrangements.

Figure 6 shows that, the equivalent $\gamma^{\prime}$ of both the parallel and serial arrangement is always higher than $\gamma_{2}^{\prime}$. For the parallel arrangement, $\gamma_{2}^{\prime} \leq \gamma^{\prime} \leq \gamma_{1}^{\prime}$, the stronger bond contributes more to the equivalent $\gamma^{\prime}$; when $\gamma_{1}^{\prime} / \gamma_{2}^{\prime}=1$, the equivalent $\gamma^{\prime}$ is independent on $K_{10} / K_{20}$. For serial configuration, $\gamma_{2}^{\prime} \leq \gamma^{\prime} \leq 2 \gamma_{1}^{\prime}$, the weaker bond contributes more to $\gamma^{\prime}$. For equivalent $\gamma^{\prime}$, according to Eq. (40), the lowest value $2 \gamma_{1}^{\prime} \gamma_{2}^{\prime} /\left(\gamma_{1}^{\prime}+\gamma_{2}^{\prime}\right)$ is achieved when $K_{10} \gamma_{1}^{\prime}=K_{20} \gamma_{2}^{\prime}$, and the mismatch of $K_{i} \gamma_{\mathrm{i}}^{\prime}$ of neighboring bonds causes an increase in the anharmonicity. To increase anharmonicity and reduce the sound velocity, the serial arrangement is preferred.

\section{PREDICTION OF THERMAL CONDUCTIVITY}

Using the relations for $\gamma, \alpha$ and $T_{D}$, when $\omega_{c}$ is comparable with $\omega_{D}$, the thermal conductivity can be readily calculated using the Slack relation. The predicted thermal conductivities of some crystals at high temperatures are listed in Table II and shown in Fig. 7(a), and the measured values and the values calculated by Slack $^{11}$ are also given. Note Slack used $T_{D, \infty}$ calculated from the phonon density of states $D_{p}$, which is different from the measured $T_{D}$ listed in Table II. Table II shows that the thermal conductivities and the Debye temperatures estimated by our model agree well with the experimental results and the Slack results, but the Grüneisen parameters are normally overestimated in our model, as discussed in Sec. IV. The average mean square error between the estimated values and the experimental results is about $20 \%$. Slack used $T_{D, \infty}$ along with the experimental $\gamma$ (but 0.7 was used for $\mathrm{Ge}, \mathrm{Si}$, and $\mathrm{SiC}$ and for better agreement with the experiments ${ }^{11}$ ), both of which are normally slightly lower than the values estimated in our model. Note that we also predict the lattice thermal conductivity of $\mathrm{Al}$ and $\mathrm{Pt}$, by only considering the phonon-phonon scattering. The crystalline metals normally have a low lattice thermal conductivity, not only due to the strong scattering of phonons by free electrons, but also due to their large Grüneisen parameters and small force constants (caused by long-range electrostatic interactions).

When $\omega_{c} \ll \omega_{D}$, the thermal transport is dominated by the short-range acoustic phonons and optical phonons. While
TABLE II. Predicted thermal conductivities and parameters of some crystals at given temperatures. The experimental results (Refs. 11 and 13) are shown in the parentheses, and the calculated results by Slack are shown in the brackets. Note that Slack used $T_{D, \infty}$ determined from the phonon density states, which is different from the experimental $T_{D}$ listed here. Also Slack used the $\gamma$ derived from experiments except diamond, $\mathrm{SiC}, \mathrm{Ge}, \mathrm{GaAs}$, and BP (he chose 0.7 for these crystals for better agreement with the experiments). The values of $m$ used in the calculations are all estimated using Eq. (36), rather than from the experimental results.

\begin{tabular}{lccccc}
\hline \hline Crystals & $T(\mathrm{~K})$ & $T_{D}$ & $N_{c}$ & $\gamma$ & $k(\mathrm{~W} / \mathrm{m} \mathrm{K})$ \\
\hline $\mathrm{Ar}$ & 84 & $94(85)$ & 1 & $2.83(2.73)$ & $0.5(0.4)[3.8]$ \\
$\mathrm{Kr}$ & 66 & $87(73)$ & 1 & $2.83(2.84)$ & $1.1(0.5)[0.4]$ \\
Diamond & 300 & $2183(2230)$ & 2 & $1.0(0.9)$ & $1292(1350)$ \\
$\mathrm{Ge}$ & 235 & $382(360)$ & 2 & $1.0(0.76)$ & $95(83)[89]$ \\
$\mathrm{Si}$ & 395 & $584(625)$ & 2 & $1.0(0.56)$ & $76.7(115)[93]$ \\
$\mathrm{Cu}$ & 300 & $339(315)$ & 1 & 2.0 & $14.4(10)^{\mathrm{b}}$ \\
$\mathrm{Pt}$ & 300 & $194(230)$ & 1 & 2.0 & $9.1\left(6^{\mathrm{b}}\right)$ \\
$\mathrm{GaAs}$ & 220 & $367(346)$ & 2 & $1.01(0.75)$ & $72(81)[77]$ \\
$\mathrm{CaF}$ & 345 & $453(510)$ & 3 & $1.89(1.89)$ & $7.0(8.5)[9.1]$ \\
$\mathrm{MgO}$ & 600 & $1034(945)$ & 2 & $1.68(1.44)$ & $53(25)[28]$ \\
$\mathrm{NaCl}$ & 230 & $382(330)$ & 2 & $1.71(1.57)$ & $11.1(8.6)[6.3]$ \\
$\mathrm{c}-\mathrm{BN}$ & 300 & 1614 & 2 & 1.22 & $733(748)$ \\
$\mathrm{SiC}$ & 300 & $1212(1079)$ & 2 & $1.11(0.76)$ & $463(490)[461]$ \\
$\mathrm{BP}^{\mathrm{a}}$ & 670 & $891(982)$ & 2 & 1.0 & $97.46(110)[166]$ \\
$\mathrm{PN}^{\mathrm{a}}$ & 300 & 890 & 2 & 1.16 & 329 \\
$\mathrm{CSe}^{\mathrm{a}}$ & 300 & 706 & 2 & 1.0 & 327 \\
\hline \hline
\end{tabular}

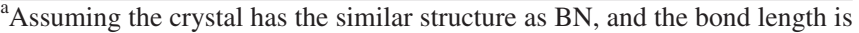
calculated from the combinative rule.

${ }^{\mathrm{b}}$ The lattice conductivities are from Ref. 10. They are obtained by subtracting the electrical thermal conductivity (derived from Wiedemann-Franz law) from the total thermal conductivity.

the acoustic contribution can be calculated using a relation similar to the Cahill-Pohl relation, ${ }^{12,29}$ the optical part is difficult to determine and it is comparable to the acoustic contribution. ${ }^{2,12}$ However, for some special atomic structures, the phonon mean-free path is limited by the crystal structure, and the thermal conductivities of such crystals often exhibit temperature-independence above the Debye temperature. According to the kinetic relation ${ }^{13} k=n_{a} c_{v} u_{p, g} \lambda / 3$ ( $c_{v}$ is the heat capacity per atom), if the phonon mean-free path $\lambda$ can be determined from the characteristics of the structure, the thermal conductivity can also be easily calculated.

There exist many special structures that can limit the phonon mean-free path at high temperature. For example, in the filled skutterudite structures, ${ }^{30}$ the fillers act as scatterers and limit the phonon mean-free path to be the distance between the scatterers. Here we discuss the cage-bridge structure, which is common for nanoporous crystals, e.g., zeolites, MOFs, and many molecular crystals.

The cage-bridge structure includes complex multiatomic cages connecting by relatively simple bridges [see Fig. 7(b)] (sometimes the cages may also be joined directly without bridges). In such a structure, the atoms in the cage are normally much more than the atoms acting as connectors. Zeolites and MOFs are good examples of such structures. Some siliceous zeolites, e.g., LTA, FAU and SOD, contain the complex sodalite cage built from $\mathrm{SiO}_{4}$ tetrahedra. ${ }^{2}$ MOF-5, the smallest cubic MOF structure, is comprised of $\mathrm{Zn}_{4} \mathrm{O}$ clusters linked by 1,4-benzenedicarboxylate (BDC). ${ }^{12}$ 
(a) $\omega_{c} \approx \omega_{\mathrm{D}}:$ Long-range acoustic phonons dominates

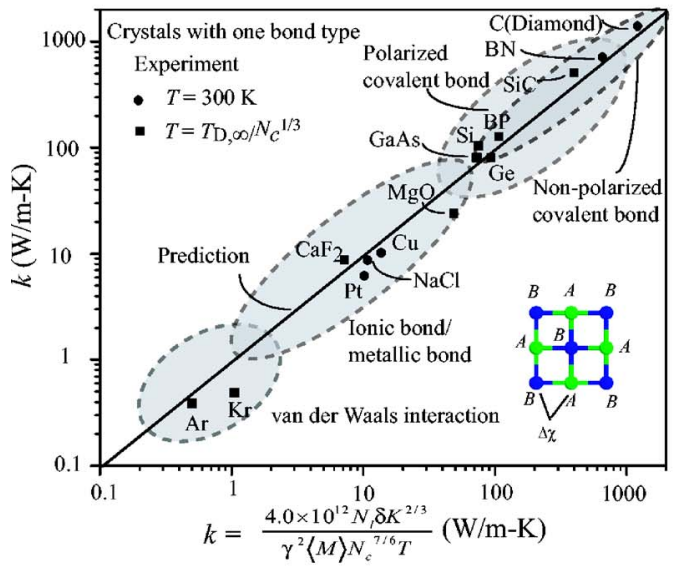

(b) $\omega_{c} \ll<\omega_{\mathrm{D}}$ : Short-range and optical phonons are important

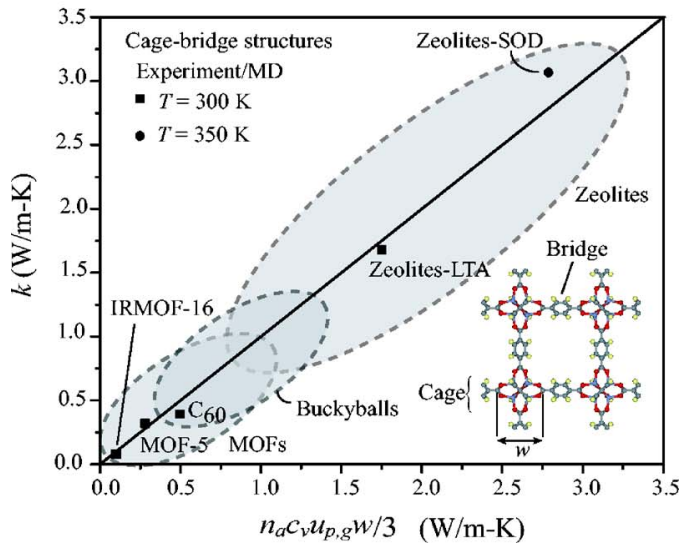

FIG. 7. (a) Comparison of the predicted lattice thermal conductivity of some compact crystals with the experimental results. (b) Comparison of the calculated thermal conductivities of some cage-bridge crystals with the experimental or MD results.

Many molecular crystals consist of large, complex molecules held together by weak van der Waals interactions or hydrogen bonds. The intramolecular interactions are much stiffer than the intermolecular interactions. They can also be considered a special type of cage-bridge structure, and each complex molecule can be considered a cage.

When $T \geq T_{D}$, all the vibration modes will have the same contribution to the total vibration energy. Since most atoms lie in the cage, most vibration energy is located in the cage and a fraction of the vibration energy transports to the next cage through connectors. However, the large coordination number difference or bond stiffness difference make the connector a bottleneck for the energy transport, and most phonon energy is localized in the cage, or reflected at the connectors. McGaughey and Kaviany ${ }^{2}$ showed that the Si-O-Si bonds contribute to the energy localization in FAU- and SOD-zeolites. Our work for MOF-5 (Ref. 12) also showed the carbon connector limits the transport of phonon energy. An indicator of this phenomena is the large difference between the phonon partial density of states (PDOS, weighted by the concentration of atoms) of the cage and that of the connectors (as shown in Ref. 12). Therefore, the connectors will act as scatterers in the structure. If the cage is relatively rigid (phonons experience little scattering within the cage),
TABLE III. Comparison of predicted thermal conductivities of some cagebridge structures calculated by Eq. (42) and the Slack relation, with the experimental and the MD results.

\begin{tabular}{lccccccc}
\hline \hline & & & & \multicolumn{3}{c}{$k(\mathrm{~W} / \mathrm{m} \mathrm{K})$} \\
\cline { 5 - 8 } \multicolumn{1}{c}{ Crystals } & $T(\mathrm{~K})$ & $u_{p, g}(\mathrm{~m} / \mathrm{s})$ & $w(\AA)$ & Eq. $(42)$ & $k_{\mathrm{CP}}$ & exp./MD \\
\hline MOF-5 $^{\mathrm{a}}$ & 300 & 1184 & 7.16 & 0.28 & 0.025 & 0.32 \\
IRMOF-16 $^{\mathrm{a}}$ & 300 & 600 & 7.16 & 0.10 & 0.01 & 0.08 \\
SOD $^{n}$ & 350 & $4200^{\mathrm{b}}$ & 8.88 & 2.79 & 1.58 & $3.09^{\mathrm{c}}$ \\
LTA & 300 & $3200^{\mathrm{b}}$ & 8.88 & 1.75 & 0.47 & $1.68^{\mathrm{c}}$ \\
$C_{60}$ & 260 & $2000^{\mathrm{d}}$ & 7.00 & 0.52 & 0.007 & $0.4^{\mathrm{e}}$ \\
\hline \hline
\end{tabular}

${ }^{\mathrm{a}}$ Values for MOF-5 are taken from Ref. 12. The data for IRMOF-16 is calculated by MD using the same potentials.

${ }^{b}$ Values are derived from the bulk modulus (Ref. 23), by setting poisson ratio as 0.3 .

${ }^{\mathrm{c}}$ Values are taken from MD results from Ref. 2.

${ }^{\mathrm{d}}$ Values are derived from the bulk modulus (Ref. 31)

${ }^{\mathrm{e}}$ Values are from Ref. 32.

the phonon mean-free path will be limited by the distance between the connectors at the boundary of the cage, which is often the same as the cage size $w$. For molecular crystals, $w$ is essentially the dimension of the molecule. Then we have

$$
k=\frac{1}{3} n_{a} c_{v} u_{p, g} w .
$$

When the temperature is higher than the Debye temperature, $c_{v}$ can be simply set as $3 k_{B}$.

This simple mean-free path model for the cage-bridge structures leads to good agreement with the experimental values or the MD results, as shown in Table III. The values calculated by the Slack relation $\left(k_{C P}\right)$ are also shown, and it is apparent that $\left(k_{C P}\right)$ has a lower value.

When the temperature decreases, the high frequency phonon modes caused by the internal vibrations of the cage will decrease much faster than the low frequency modes, and the fraction of localized energy will decrease. When the temperature is much lower than the Debye temperature, the fraction of localized vibration energy will be small and the phonon mean-free path will no longer be limited by the cage size.

\section{SUMMARY AND CONCLUSION}

A simple atomic structure based model for the estimation of lattice thermal conductivity of crystals at moderate and high temperature is proposed. When the critical frequency $\omega_{c}$ is comparable with the Debye frequency $\omega_{D}$, the thermal conductivity is obtained on the basis of the Debye temperature and the Grüneisen parameter calculated from the atomic configuration of the structure.

The calculation of the Debye temperature involves three steps: (i) estimation of the force constant of arbitrary pair of interacting atoms on the basis of a phenomenological combinative rule and data listed in Table I; (ii) simplification of the network system of bonds using analogy with a spring system, and calculation of the equivalent bonds; (iii) calculation of the Debye temperature using the equivalent bonds and the topology of the crystal.

The determination of the Grüneisen parameter consists two steps: (i) Estimation of the bond-scaling parameter of 
each single bond; (ii) estimation of the equivalent Grüneisen parameter using Eqs. (38) and (40) on the basis of the configuration of bonds. It is found that when $K \gamma^{\prime}$ of the bonds match, the equivalent Grüneisen parameter achieves its minimum.

This simple atomic structure-based model can be used to quickly estimate the high-temperature thermal conductivity of crystals. On the other hand, some useful insights into the design of materials with desired properties can be extracted.

According to Eqs. (1) and (26), we have

$$
k=\frac{4.0 \times 10^{12} N_{l} K^{3 / 2} \delta}{\gamma^{2}\langle M\rangle^{1 / 2} N_{c}^{7 / 6} T},
$$

where $N_{l}$ is a constant related only to lattice type. Therefore, to increase the thermal conductivity, one may increase the equivalent force constant $K$ and lattice constant $a$, while reducing the mean atomic weight $\langle M\rangle, N_{c}$, and the Grüneisen parameter $\gamma$. Here FCC is expected to achieve a high thermal conductivity. The opposite approaches can be used to achieve a low lattice thermal conductivity.

Evidently, molecular crystals will normally have a very low thermal conductivity because of the small $K$, large $\gamma$, and $N_{c}$.

For ionic and covalent diatomic crystals, $\quad \gamma=2$ $-\exp \left(-\Delta \chi^{2} / 4\right)$, then Eq. (44) can be rewritten as

$$
k=\frac{4.0 \times 10^{12} N_{l} \delta K^{3 / 2}}{\left[2-\exp \left(-\Delta \chi^{2} / 4\right)\right]^{2}\langle M\rangle^{1 / 2} N_{c}^{7 / 6}} .
$$

For compact structures, $\delta$ may be set as the mean diameter of the atoms.

Table I and Fig. 1(a) show that most metals have a low $K_{A A, 1}$ around $50 \mathrm{~N} / \mathrm{m}$. Even when they bond with $\mathrm{F}$ (which has the highest $\left.K_{A A, 1}\right), K_{A B, 1}$ is expected to be lower than $250 \mathrm{~N} / \mathrm{m}$. Also, metal elements normally have a heavy mass and ionic bonds have a relatively high $\Delta \chi$. In comparison, covalent bonds may have a higher $K_{A B, 1}$, lower $\Delta \chi$, and those nonmetallic elements with a high $K_{A A, 1}$ have a relatively light mass. Thus, for high thermal conductivity, covalent crystals are preferred. Among covalent crystals, the compounds of $\mathrm{N}$ and $\mathrm{C}$ are expected to have a high thermal conductivity, since $\mathrm{N}$ and $\mathrm{C}$ have the highest $K_{A A, 1}$, moderate $\chi$, light masses, and possibly high bond orders. In general, the sequence of lattice conductivity for crystals is $k$ (nonpolarized covalent crystal) $>k$ (polarized covalent crystal) $>k$ (ionic crystal) $>k$ (molecular crystal), as shown in Fig. 7(a). Furthermore, the oxidation states of the elements need to match and the mass difference should be small to achieve a small $N_{c}$. Materials satisfying these conditions are expected to have a high thermal conductivity, e.g., BN, AlN, BP, and SiC, furthermore, it is expected that PN and CSe would have a high thermal conductivity if they could be synthesized (listed in Table II).

For thermoelectic materials or thermal insulators, lower phonon thermal conductivity is desired. In addition to the common strategies (e.g., using heavy atoms and making a complex unit cell), the following may be used as a guide:

(i) Adding flexible structures on the transport path. Flexible structures with bending or torsion motion often have a small $K$ and high $\gamma$, thus they can both reduce the sound velocity and increasing the anharmonic scattering.

(ii) Making the bonds as perpendicular as possible to the transport path. This can reduce the projection of the force constant, and lower the Debye temperature.

(iii) Enlarging the mismatch of $K \gamma$ of the neighboring bonds. Substituting some bonds with bonds with higher $\gamma$, or substituting some bonds to increase the mismatch of force constant, can increase the anharmonic scattering. For example, by replacing some covalent pairs with pairs with charges or larger electronegativity difference, the phonon thermal conductivity can be reduced, as observed by Cahill et al. $^{29}$

(iv) Arranging the bonds with high $\gamma$ in series. This will help increase the anharmonic scattering.

For special cage-bridge structures, a simple mean-free path model based on the kinetic theory is proposed, which indicates that at high temperatures, the mean-free path of cage-bridge structure is equal to the dimension of the cage. This simple model shows good agreement with experimental and the MD results.

The simple atomic structure model proposed in this paper allows for the quick estimation of thermal transport properties, and can be used as a guide for the design of new materials with a desired lattice conductivity.

\section{ACKNOWLEDGMENTS}

This work has been supported by the U.S. Department of Energy, Office of Basic Energy Sciences under Grant No. DE-FG02-00ER45851. The authors would like to thank Alan McGaughey and Xiulin Ruan for their insightful comments and constructive discussions.

\section{APPENDIX: GRÜNEISEN PARAMETER FOR LJ OSCILLATORS}

For LJ oscillators, the vibrational energy $E_{l}$ and the mean atomic separation $\left\langle r_{l}\right\rangle$ of the motion with the principle quantum number $l$ of this oscillator can be expressed as ${ }^{27}$

$$
\begin{aligned}
& E_{l}=\hbar \omega\left(l+\frac{1}{2}\right)-C_{e} \hbar^{2} \omega^{2}\left(l+\frac{1}{2}\right)^{2}, C_{e}=\frac{5(m+n+3)^{2}}{48 \varphi_{o} m n}, \\
& \left\langle r_{l}\right\rangle=r_{o}+C_{r} r_{o} \hbar \omega\left(l+\frac{1}{2}\right), \quad C_{r}=\frac{3(m+n+3)}{2 \varphi_{o} m n},
\end{aligned}
$$

where $-\varphi_{o}$ is the equilibrium potential energy.

The statistical mean vibration energy $E(\omega)$ and interatomic separation $\bar{r}(\omega)$ for frequency $\omega$ can then be given $\operatorname{as}^{26}$

$$
\begin{aligned}
& E(\omega)=F-T\left(\frac{\partial F}{\partial T}\right)_{V}, F=-\hbar \omega \ln Z, \\
& \bar{r}(\omega)=\frac{\sum_{l=0}^{\infty}\left\langle r_{l}\right\rangle e^{-E_{l} / k_{B} T}}{\sum_{l=0}^{\infty} e^{-E_{l} / k_{B} T}}=r_{o}+\frac{C_{r} r_{o} \hbar \omega}{2}+\frac{C_{r} r_{o} \hbar \omega}{e^{\hbar \omega / \hbar \omega}-1},
\end{aligned}
$$

where $Z=\sum_{l=0}^{\infty} e^{-E_{l} / k_{B} T}$, is the partition function of the oscillator. Using the Debye approximation, the total vibrational energy $E$ can be given as 


$$
\begin{aligned}
& E=\int_{0}^{\omega_{D}} E(\omega) D_{p}(\omega) d \omega \\
& \simeq E_{o}+9 N k_{B} T x_{D}^{-3} f\left(x_{D}\right)-9 C_{e} N\left(k_{B} T\right)^{2} x_{D}^{-3} f_{1}\left(x_{D}\right), \\
& f\left(x_{D}\right)=\int_{0}^{x_{D}} \frac{x^{3} \mathrm{~d} x}{e^{x}-1}, x_{D}=T_{D} / T \\
& f_{1}\left(x_{D}\right)=\int_{0}^{x_{D}} \frac{x^{4}\left(1+e^{x}\right) \mathrm{d} x}{\left(e^{x}-1\right)^{2}},
\end{aligned}
$$

where $D_{p}(\omega)$ is the phonon density of states, $\omega_{D}$ is the Debye frequency, and $E_{o}$ is the zero point energy.

Similarly, the mean interatomic separation $r_{e}$ can be written as

$r_{e}=\frac{1}{3 N} \int_{0}^{\omega_{D}} D_{p}(\omega) \bar{r}(\omega) \mathrm{d} \omega=r_{o}+r_{1}+3 k_{B} T C_{r} r_{o}\left(\frac{T}{T_{D}}\right)^{3} f_{1}\left(x_{D}\right)$,

where $r_{1}$ is the deviation due to the zero-point vibration, and $r_{2}$ is related to the thermal vibration and anharmonicity of the potential. $r_{e, o}=r_{o}+r_{1}$ is the equilibrium separation at $T$ $=0 \mathrm{~K}$.

The Mie-Grüneisen equation of state is ${ }^{26}$

$$
p+\frac{\mathrm{d} U}{\mathrm{~d} V}=\gamma \frac{E_{v}}{V}
$$

where $p$ is the pressure, $U$ is the lattice potential energy, $E_{v}$ is the total vibration energy excluding the zero-point energy $E_{o}$, and $V$ is the volume.

For small perturbation

$$
\frac{\mathrm{d} U}{\mathrm{~d} V} \simeq B_{o} \frac{V-V_{o}}{V_{o}}=3 B_{o} \frac{r-r_{o}}{r_{o}},
$$

where $B_{o}$ is the bulk modulus at zero temperature, and $V_{o}$ is the initial volume. Since $B_{o}=U_{o} m n /\left(9 V_{o}\right)$ (Ref. 26) and $\gamma$ $=\gamma^{\prime}$ (the crystal contains only one bond type), by setting $r_{e, o}=r_{o}$, Eqs. (A4), (A5), and (A7) give

$$
\gamma^{\prime} \simeq \gamma_{\mathrm{o}}^{\prime}\left[1+C_{e} k_{B} T \frac{f_{1}\left(x_{D}\right)}{f\left(x_{D}\right)}\right], \quad \gamma_{\mathrm{o}}^{\prime}=\frac{m+n+3}{6} .
$$

${ }^{1}$ A. Griesinger, K. Spindler, and E. Hahne, Int. J. Heat Mass Transfer 42, 4363 (1999).

${ }^{2}$ A. J. H. McGaughey and M. Kaviany, Int. J. Heat Mass Transfer 47, 1799 (2004).

${ }^{3}$ P. K. Schelling, S. R. Phillpot, and P. Keblinski, Phys. Rev. B 65, 144306 (2002).

${ }^{4}$ J. Che, T. Cagin, W. Deng, and W. A. Goddard, J. Chem. Phys. 113, 6888 (2000).

${ }^{5}$ A. R. Leach, Molecular Modeling Principles and Applications (Addison Wesley Longman, Reading, MA, 1996).

${ }^{6}$ J. Callaway, Phys. Rev. 113, 1046 (1959).

${ }^{7}$ M. G. Holland, Phys. Rev. 132, 2461 (1963).

${ }^{8}$ A. J. H. McGaughey and M. Kaviany, Phys. Rev. B 69, 094303 (2004).

${ }^{9}$ C. L. Julian, Phys. Rev. 137, A128 (1965).

${ }^{10}$ R. Berman, Thermal Conduction in Solids (Oxford University Press, New York, 1976).

${ }^{11}$ G. A. Slack, Solid State Phys. 34, 1 (1979).

${ }^{12}$ B. L. Huang, A. Mcgaughey, and M. Kaviany, Int. J. Heat Mass Transfer (2006) (in press).

${ }^{13}$ N. W. Ashcroft and N. D. Mermin, Solid State Physics (Saunder College Press, Philadelphia, 1976).

${ }^{14}$ G. Herzberg, Molecular Spectra and Molecular Structure II. Infrared and Raman Spectra of Polyatomic Molecules (Van Nostrand, New York, 1945).

${ }^{15}$ J. P. Biersack and J. F. Ziegler, Nucl. Instrum. Methods Phys. Res. 194, 93 (1982).

${ }^{16}$ I. Feranchuk, A. Minkevich, and A. Ulyanenkov, Eur. Phys. J.: Appl. Phys. 19, 95 (2002).

${ }^{17}$ E. B. Wilson, J. C. Decius, and P. C. Cross, Molecular Vibrations: The Theory of Infrared and Raman Vibrational Spectra (Van Nostrand, New York, 1955).

${ }^{18}$ J. Paolini, J. Comput. Chem. 11, 1160 (1990).

${ }^{19}$ G. Herzberg, Molecular Spectra and Molecular Structure IV. Constants of Diatomic Molecules (Van Nostrand, New York, 1979).

${ }^{20}$ L. Pauling, The Nature of Chemical Bonds (Cornell University Press, Ithaca, 1960).

${ }^{21}$ C. Kittel, Introduction to Solid State Physics (Wiley, New York, 1996).

${ }^{22}$ D. Sirdeshmukh, P. Krishna, and K. Subhadra, J. Mater. Sci. 38, 2001 (2003).

${ }^{23}$ R. Astala, S. M. Auerbach, and P. A. Monson, Phys. Rev. B 71, 014112 (2005).

${ }^{24}$ M. T. Dove, Introduction to Lattice Dynamics (Cambridge University Press, Cambridge, Massachusetts, 1993).

${ }^{25}$ R. Zallen and M. Slade, Phys. Rev. B 18, 5775 (1978).

${ }^{26}$ A. R. Ruffa, J. Chem. Phys. 83, 6405 (1985).

${ }^{27}$ F. G. Mateo, J. Zuniga, A. Requena, and A. Hidalgo, J. Phys. B 23, 2771 (1990).

${ }^{28}$ H. Septzler, J. Phys. Chem. Solids 33, 1727 (1972).

${ }^{29}$ D. G. Cahill, S. K. Watson, and R. O. Pohl, Phys. Rev. B 46, 6131 (1992).

${ }^{30}$ C. Uher, Semicond. Semimetals 69, 140 (2001).

${ }^{31}$ S. Duclos, K. Brister, R. Haddon, A. Kortan, and F. Thiel, Nature 351, 380 (1991).

${ }^{32}$ R. Yu, N. Tea, M. B. Salamon, D. Lorents, and R. Malhotra, Phys. Rev. Lett. 68, 2050 (1992). 